\title{
Self-optimization of coverage and capacity based on a fuzzy neural network with cooperative reinforcement learning
}

\author{
Shaoshuai Fan ${ }^{1}$, Hui $\operatorname{Tian}^{1 *}$ and Cigdem Sengul ${ }^{2}$
}

\begin{abstract}
Self-organization is a key concept in long-term evolution (LTE) systems to reduce capital and operational expenditures (CAPEX and OPEX). Self-optimization of coverage and capacity, which allows the system to periodically and automatically adjust the key radio frequency (RF) parameters through intelligent algorithms, is one of the most important tasks in the context of self-organizing networks (SON). In this paper, we propose self-optimization of antenna tilt and power using a fuzzy neural network optimization based on reinforcement learning (RL-FNN). In our approach, a central control mechanism enables cooperation-based learning by allowing distributed SON entities to share their optimization experience, represented as the parameters of learning method. Specifically, SON entities use cooperative Q-learning and reinforced back-propagation method to acquire and adjust their optimization experience. To evaluate the coverage and capacity performance of RL-FNN, we analyze cell-edge performance and cell-center performance indicators jointly across neighboring cells and specifically consider the difference in load distribution in a given region. The simulation results show that RL-FNN performs significantly better than the best fixed configuration proposed in the literature. Furthermore, this is achieved with significantly lower energy consumption. Finally, since each self-optimization round completes in less than a minute, RL-FNN can meet the need of practical applications of self-optimization in a dynamic environment.
\end{abstract}

Keywords: LTE; Self-organizing network; Coverage and capacity; Fuzzy neural network; Reinforcement learning

\section{Introduction}

Today's cellular radio technologies are developed to operate closer to Shannon capacity bound. Yet, efficient PHY layer solutions may not necessarily translate to efficient resource utilization as the network performance relies on the dynamics of the radio network environment [1]. To this end, one of the key concepts of the prominent 4G technology, long-term evolution (LTE), is self-organizing network (SON), which is expected to help LTE cope with network and traffic dynamics both during deployment and operation [2]. Essentially, SON, which enables self-configuration, self-optimization, and self-healing, is expected to improve network performance and user

\footnotetext{
*Correspondence: Tianhui@bupt.edu.cn

1 State Key Laboratory of Networking and Switching Technology, Beijing University of Posts and Telecommunications, Beijing 100876, China Full list of author information is available at the end of the article
}

quality of experience while reducing capital expenditures (CAPEX) and operational expenditures (OPEX) [3-7].

Coverage and capacity optimization (CCO) is one of the typical operational tasks of SON $[2,8]$. CCO allows the system to periodically adjust to the changes in traffic (i.e., load and location) and the radio environment by adjusting the key radio frequency (RF) parameters (e.g., antenna configuration and power) through intelligent algorithms. For the online $\mathrm{CCO}$ problem, there is no definite mapping function from the inputs and parameters to be adjusted to the coverage and capacity performance. The main reason is the complexity of adjusting all the configuration parameters affecting both coverage and capacity. In addition, the configuration parameter space is too large, which prohibits exhaustive search [9]. Thereby, most algorithms are designed in a heuristic way.

Among the existing approaches, the artificial intelligence-based approaches that accumulate the operational

\section{望 Springer}

(c) 2014 Fan et al.: licensee Springer. This is an Open Access article distributed under the terms of the Creative Commons Attribution License (http://creativecommons.org/licenses/by/2.0), which permits unrestricted use, distribution, and reproduction in any medium, provided the original work is properly credited. 
and optimization experience and form optimization policies based on these experiences are the most promising. The optimization experience, which is shared among SON entities, constitutes traffic and radio conditions, and current configuration and performance. Current configuration is typically defined based on a single optimization parameter (e.g., antenna tilt) and does not take into account the impact of other parameters such as power in optimizing capacity and coverage. Also, learning is typically performed in a selfish manner without considering the learning cooperation of all the entities.

To overcome the aforementioned problems, in this paper, we design a distributed fuzzy neural network (FNN) to guide the joint optimization of coverage and capacity. Our solution introduces a central control mechanism to facilitate cooperative learning by sharing the optimization experience of SON entities. As the core part of the fuzzy neural network, both fuzzy inference rule base and parameters of membership functions are acquired and adapted with reinforcement learning method which can achieve a predefined goal by directly interacting with its uncertain environment and by properly utilizing past experience derived from previous actions. To control coverage and capacity, we jointly optimize antenna tilt and power while considering the varied and non-uniform traffic load distribution of the cells within a specific region.

The rest of the paper is organized as follows. In Section 2, we describe related research in the literature. In Section 3, we discuss the factors that affect our approach and describe the architecture for CCO. In Section 4, we present the details of the proposed reinforcement learning-based FNN optimization (RL-FNN) executed in distributed SON entities. In Section 5, we present the simulation results. Finally, we draw the conclusions in Section 6.

\section{Related work}

Majority of the coverage and capacity optimization algorithms are heuristic due the complexity of the problem. For instance, local search methods, such as gradient ascent [10] and simulated annealing [11,12], are adopted for radio network planning. In [10], a heuristic variant of the gradient ascent method was adopted to optimize antenna tilt. In $[11,12]$, simulated annealing algorithm was used to control the downlink transmit power. The proposed approaches rely on the accurate downlink interference information in an eNodeb's own and neighboring cells under all possible parameter configurations. However, such information can hardly be predicted due to not having a precise mapping from parameter adjustments to the current performance. Taguchi method, which is superior to the local search methods in exploring the search space, was used in [13] to optimize radio network parameters. Yet, a great number of experiments are needed to explore the large parameter space and determine the impact of different parameter values on the network performance during operation. Finally, in all these algorithms, each iteration step caused by the dynamics in traffic and the radio environment is a trial-and-error process. Due to the risk of negative performance impact, trial-and-error is prohibitive in real networks.

To prevent potential drastic changes in the network performance, the artificial intelligence approach, which can accumulate the operational and optimization experience and form optimization policies based on the experience, has significant potential [14-17]. In [1,18], a case-based reasoning algorithm enables distributed decision-making. The algorithm stores past successful optimization instances that improved the performance in the memory and applies these instances directly to new situations. In [19-21], a fuzzy Q-learning algorithm was used to learn the optimal antenna tilt control policy based on the continuous inputs of current antenna configuration and corresponding performance, and output of the optimized antenna configuration. Yet, the impact on neighboring cells due to such an adjustment was neglected. To overcome the suboptimal performance of selfish learning, the approaches proposed in $[6,22]$ permit the cells to share their performance statistics with their neighbor cells so that each SON entity tries to learn the optimal action policy based on the overall reward of the neighborhood instead of local selfish rewards. However, the potential from having SON entities learn cooperatively was not taken into consideration. Also, the fuzzy membership functions in the proposed fuzzy Q-learning algorithms were predefined by intuition or partial operation experience, which may affect the optimization performance. Moreover, in contrast to our approach, these approaches only optimize the antenna tilt.

\section{Coverage and capacity optimization under hybrid SON architecture}

To achieve capacity and coverage optimization (CCO), we adopt the hybrid architecture shown in Figure 1. On one hand, each site has SON entities corresponding to each sector (three sectors in our study), and each entity runs its optimization process in a distributed manner which can provide fast adaptation. On the other hand, a central entity located in the network management system (NMS) is needed whose main function, in our proposed approach, is to realize the cooperation of distributed optimization by collecting optimization experience of all distributed entities and sharing them in a global manner.

In our system, the goal of $\mathrm{CCO}$ problem is the joint optimization of coverage and capacity by automatically 


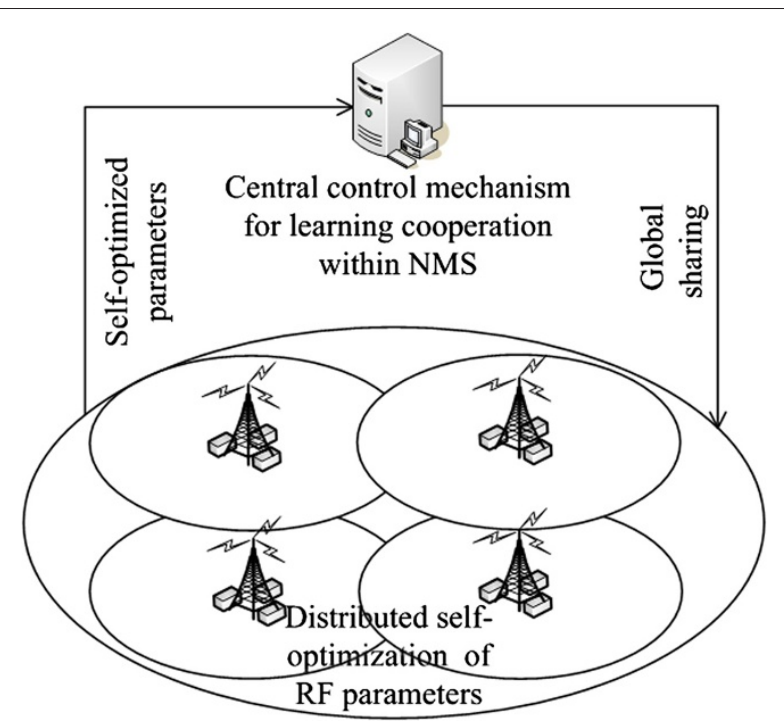

Figure 1 Hybrid SON architecture for CCO.

adjusting the RF parameters which affect downlink signal to interference plus noise ratio (SINR):

$$
\operatorname{SINR}_{i j}=\frac{P_{i} G_{i j}}{N+\sum_{k \neq i} P_{k} G_{k j}},
$$

where $P_{i}$ is the transmit power on each resource block (RB) which is in direct proportion to the total power of eNodeB $i$ if the power is equally allocated, $N$ is the received noise, and $G_{i j}$ is the link loss from eNodeB $i$ to user $j$ including path loss, antenna gain, shadowing, multipath fading, etc.

Antenna configuration and total power are the key RF parameters for optimization. For 3D antenna configuration, there are four important parameters that can be adjusted: antenna azimuth $\varphi_{a s}$, horizontal half-power beam width $\varphi_{3 d B}$, vertical half-power beam width $\theta_{3 d B}$, and remote electrical tilt (RET) $\theta_{\text {gtilt }}$. The antenna configuration parameters affect the three-dimensional antenna gain $A(\varphi, \theta)$ as follows [23]:

$$
A(\varphi, \theta)=-\min \left\{-\left[A_{\mathrm{H}}(\varphi)+A_{\mathrm{V}}(\theta)\right], A_{m}\right\} .
$$

The horizontal antenna gain and vertical antenna gain are defined as

$$
\begin{aligned}
& A_{\mathrm{H}}(\varphi)=-\min \left[12\left(\frac{\varphi-\varphi_{a s}}{\varphi_{3 d B}}\right)^{2}, A_{m}\right] \\
& A_{\mathrm{V}}(\theta)=-\min \left[12\left(\frac{\theta-\theta_{\text {gtilt }}}{\theta_{3 d B}}\right)^{2}, \mathrm{SLA}_{v}\right] .
\end{aligned}
$$

In Equations 3 and $4, A_{m}$ denotes the maximum antenna front-to-back attenuation and $\mathrm{SLA}_{v}$ denotes the maximum vertical slant angle attenuation.

While the RET optimization can provide larger gains in terms of cell-edge and cell-center performance [1], power optimization can improve coverage and capacity performance and also power efficiency to some extent. Considering these factors, the antenna tilt and total power are chosen as the parameters to adjust in order to improve the coverage and capacity performance.

Many factors should be considered while adjusting the RF parameters, including cell-edge performance, cellcenter performance, inter-cell interference, and traffic load distribution. For instance, a higher value of antenna tilt or a lower value of power may result in coverage outage at the cell-edge but will also result in less inter-cell interference. On the contrary, a lower antenna tilt or a higher power may result in expansion of coverage and improvement of cell-edge performance with the risk of more inter-cell interference. Additionally, the expanded coverage of a cell may relieve the traffic load in neighboring cells at the risk of causing congestion in the expanded cell. Consequently, the fact that all the adjustments have both negative and positive consequences makes this optimization problem very complex to solve.

However, note that regardless of the underlying reason, all adjustments will affect the throughput of users. Therefore, we adopt throughput as the metric for evaluating system performance. Obviously, there is a trade-off between coverage and capacity. Typically, more coverage results in less capacity due to deteriorating signal power, and one cannot optimize both coverage and capacity at the same time. To achieve a trade-off, we define the key performance indicator (KPI) of an optimization area, e.g., sector $i$, as

$$
\mathrm{KPI}_{i}=\omega T_{i 5 \%}+(1-\omega) T_{i 50 \%} .
$$

In Equation 5, $T_{i 5 \%}$ represents cell-edge coverage, which is computed as the throughput cumulative distribution function (CDF) of $5 \%$ tile, and $T_{i 50 \%}$ denotes the cellcenter capacity, computed as the throughput CDF of $50 \%$ tile. Also, a weight factor $\omega$ is used to balance coverage and capacity performance. In this paper, we give more weight to the coverage performance as it affects user experience more.

The adjusting of the RF parameters will also have an impact on neighboring regions. Hence, the KPI of the neighbor regions should also be taken into account during the optimization. Then, the coverage and capacity optimization problem can be formulated as a joint KPI maximization problem for a region $i$ :

$$
\max _{P, \theta} \mathrm{JKPI}_{i}=\alpha \mathrm{KPI}_{i}+\frac{(1-\alpha)}{|N(i)|} \sum_{j \in N(i)} \mathrm{KPI}_{j},
$$

where $N(i)$ is the set of its neighbor areas, and $\alpha$ is the weight factor used to measure the importance of the sector's performance and its neighbors'. Note that this optimization problem is constrained by the maximum power and tilt. Additionally, the various settings of the weights (i.e., $\omega$ and $\alpha$ ) will affect the optimization target instead 
of the optimization ability. Note that the setting of these weights depends on the network operators' optimization targets and strategies. Finally, as each SON entity solves the $\mathrm{CCO}$ problem locally, we assume that the KPI information, needed from the neighboring cells, can be easily transferred via LTE X2 interfaces between eNodeBs.

\section{Fuzzy neural network with cooperative Q-learning}

In this paper, our main goal is to enable all SON entities to take simultaneous actions periodically to optimize RF parameters and learn from each other's optimization experience. In order to achieve this, we propose using a distributed Q-learning based fuzzy neural network algorithm, which we present in detail in this section.

\subsection{Architecture of RL-FNN}

The proposed RL-FNN is similar in architecture to $[24,25]$ and consists of five layers. Figure 2 shows each layer, where layer 1 consists of the input nodes, layer 2 consists of the rule antecedent nodes, layer 3 consists of the rule nodes, layer 4 consists of the rule consequent nodes, and layer 5 consists of the output nodes.

The proposed RL-FNN has two generic processes: forward operation and learning. It describes the current state based on the current power and tilt configuration, and the corresponding coverage and capacity performance are taken into account in every forward operation process to obtain the best RF parameters. RL-FNN performs the mapping function of current state to the best RF configuration in the forward operation process, while the mapping function is formed by the learning process. However, considering that the perfect input-output training sample pairs can hardly be acquired in a realistic network, reinforcement learning methods $[26,27]$ are needed for training the fuzzy neural network.

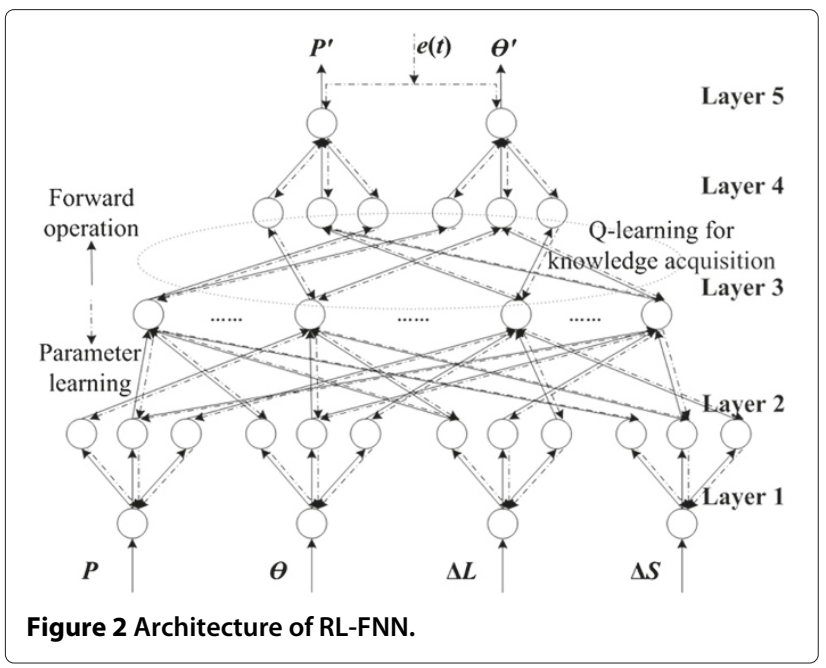

In addition, we have to keep in mind that in certain applications, the performance of fuzzy neural network highly depends on the fuzzy inference rule base and the particular membership functions, which can strongly affect the performance. Therefore, RL-FNN adopts a two-phase learning process: knowledge acquisition and parameter learning. In the knowledge acquisition phase, cooperative Q-learning method is adopted to acquire the fuzzy inference rule base. In the parameter learning phase, we adopt the reinforced back-propagation method to adjust the parameters of fuzzy membership functions. The details of the forward operation process and the twophase learning process are explained in the rest of this section.

\subsection{Forward operation \\ 4.2.1 Layer 1}

In layer 1, the input of the RL-FNN is the current RF configuration and the corresponding performance. The RF configuration is described by the current power $P$ and tilt $\theta$, and the current performance is described by JKPI (see Equation 6). Note that JKPI is not only affected by the spectrum efficiency which is determined by the RF configuration, but also affected by the load distribution (e.g., the higher the traffic load, the lower the average allocated bandwidth and throughput of users). Therefore, we adopt two parameters to describe the current state: (1) the traffic load gap $\Delta L$, which reflects the variation of load distribution among neighboring cells, and (2) the spectrum efficiency indicator gap $\Delta S$, which reflects variation in coverage and capacity performance without the effect of the traffic load. More specifically, $\Delta L$ represents the normalized difference between traffic load $L$ of the sector and the average load $\bar{L}$ of its neighboring sectors:

$$
\Delta L=\frac{L-\bar{L}}{\bar{L}} .
$$

$\Delta S$ represents the normalized difference between coverage and capacity performance indicator $S$ of the sector and the average performance $\bar{S}$ of its neighboring sectors:

$$
\Delta S=\frac{S-\bar{S}}{\bar{S}}
$$

Similar to the definition of KPI, $S=\omega S_{5 \%}+(1-\omega) S_{50 \%}$, where $S_{5 \%}$ denotes the spectrum efficiency CDF of $5 \%$ tile and $S_{50 \%}$ denotes the spectrum efficiency CDF of $50 \%$ tile.

With these two parameters, we are able to describe the current state more specifically and make more appropriate optimization decisions. So, totally we have four variables $x=(P, \theta, \Delta L, \Delta S)$ that serve as inputs to the RL-FNN. Consequently, this layer consists of four nodes 
corresponding to four input variables. The input $I_{i}^{(1)}$ and output $O_{i}^{(1)}$ of node $N_{i}^{(1)}$ in layer 1 are defined as

$$
O_{i}^{(1)}=I_{i}^{(1)}=x_{i} .
$$

\subsubsection{Layer 2}

In this layer, each input variable is fuzzified into three linguistic levels - high (H), medium (M), and low (L) - which are also called Gaussian fuzzy sets. So, this layer consists of 12 nodes. Each rule antecedent node $N_{i j}^{(2)}$ calculates the degree of membership for the $j$ th Gaussian fuzzy set associated with the $i$ th input variable. The input $I_{i j}^{(2)}$ and output $O_{i j}^{(2)}$ of node $N_{i j}^{(2)}$ in layer 2 are defined as

$$
\begin{aligned}
& I_{i j}^{(2)}=O_{i}^{(1)} \\
& O_{i j}^{(2)}=\mu_{i j}\left(I_{i j}^{(2)}\right)=\exp \left(-\left(\frac{O_{i}^{(1)}-c_{i j}^{(2)}}{\sigma_{i j}^{(2)}}\right)^{2}\right) .
\end{aligned}
$$

Here, $c_{i j}^{(2)}$ and $\sigma_{i j}^{(2)}$ are, respectively, the mean and the standard deviation of the Gaussian membership function of layer 2 .

\subsubsection{Layer 3}

Each rule node $N_{k}^{(3)}$ in this layer represents a possible IF part of an IF-THEN fuzzy inference rule and function to compute the overall degree of similarity between the observed inputs and the antecedent conditions of the $k$ th fuzzy inference rule. This layer consists of 81 nodes corresponding to 81 possible combinations of the linguistic inputs. The input $I_{k}^{(3)}$ and output $O_{k}^{(3)}$ of node $N_{k}^{(3)}$ in layer 3 are defined as

$$
\begin{aligned}
& I_{k}^{(3)}=\left(O_{1 k_{1}}^{(2)}, O_{2 k_{2}}^{(2)}, O_{3 k_{3}}^{(2)}, O_{4 k_{4}}^{(2)}\right) \\
& O_{k}^{(3)}=O_{1 k_{1}}^{(2)} \times O_{2 k_{2}}^{(2)} \times O_{3 k_{3}}^{(2)} \times O_{4 k_{4}}^{(2)} .
\end{aligned}
$$

Here, $O_{i k_{i}}^{(2)}$ is the degree of membership of the $i$ th linguistic input for the IF part of the $k$ th fuzzy inference rule.

\subsubsection{Layer 4}

Each of the two output variables of RL-FNN is fuzzified into three linguistic levels - high $(\mathrm{H})$, medium $(\mathrm{M})$, and low (L). So, this layer consists of six nodes. The links from layer 3 nodes to layer 4 nodes denote the THEN part of the IF-THEN fuzzy inference rules. The method of establishing the fuzzy inference rule base will be described in Section 4.3.

The rule consequent node $N_{l m}^{(4)}$ in layer 4 performs consequent derivation using the set of IF-THEN fuzzy inference rules. Each rule consequent node sums the output of the layer 3 nodes, which see this layer 4 node as a consequence, to identify the degree of membership for the consequent part of the rule. The input $I_{l m}^{(4)}$ and output $O_{l m}^{(4)}$ of node $N_{l m}^{(4)}$ in layer 4 are defined as

$$
\begin{aligned}
& I_{l m}^{(4)}=\left(O_{1_{l m}}^{(3)}, \cdots, O_{r_{l m}}^{(3)}\right) \\
& O_{l m}^{(4)}=O_{1_{l m}}^{(3)}+\cdots+O_{r_{l m}}^{(3)} .
\end{aligned}
$$

Here, $O_{n_{l m}}^{(3)}$ is the $n$th input to node $N_{l m}^{(4)}$ for the $m$ th Gaussian fuzzy set associated with the $l$ th output variable.

\subsubsection{Layer 5}

The best configuration value of power $P^{\prime}$ and tilt $\theta^{\prime}$ for current state is obtained in this layer as the outputs of RLFNN. This layer consists of two output nodes corresponding to two output variables $\left(P^{\prime}, \theta^{\prime}\right)$. The output nodes finally perform defuzzification of the overall inferred output fuzzy set according to the center of area method. The input $I_{l}^{(5)}$ and output $O_{l}^{(5)}$ of node $N_{l}^{(5)}$ in layer 5 are defined as

$$
\begin{aligned}
& I_{l}^{(5)}=\left(O_{l 1}^{(4)}, O_{l 2}^{(4)}, O_{l 3}^{(4)}\right) \\
& O_{l}^{(5)}=\frac{\sum_{m=1}^{3} O_{l m}^{(4)} c_{l m}^{(5)} \sigma_{l m}^{(5)}}{\sum_{m=1}^{3} O_{l m}^{(5)} \sigma_{l m}^{(5)}} .
\end{aligned}
$$

Here, $c_{l m}^{(5)}$ and $\sigma_{l m}^{(5)}$ are, respectively, the mean and the standard deviation of the Gaussian membership function of layer 5 . The method of acquiring the Gaussian membership function parameters of layer 2 and layer 5 will be described in Section 4.4.

\subsection{Q-learning for knowledge acquisition}

In order to achieve self-optimization, each entity in each cell must know what parameter tuning action should be done according to the current operation state which is determined by $x=(P, \theta, \Delta L, \Delta S)$ (defined in Section 4.2). However, it is hard to populate the fuzzy inference rule base, as the complete and accurate knowledge of the network operation can hardly be acquired online, and typically, not enough operational experience can be collected beforehand in such a complex optimization scenario. Therefore, in our approach, cooperative Q-learning algorithm is used for knowledge acquisition.

For each rule, there are nine possible inference results corresponding to the combinations of the linguistic outputs. The possible results of $k$ th rule in Q-learning are shown as

IF current state is the antecedent state of the $k$ th rule THEN $P^{\prime}$ is high and $\theta^{\prime}$ is high with $q_{k 1}$, or $P^{\prime}$ is high and $\theta^{\prime}$ is medium with $q_{k 2}$,

$$
\text { or } P^{\prime} \text { is low and } \theta^{\prime} \text { is low with } q_{k} \text {. }
$$


Here $q_{k i}$ represents the elementary quality of the $i$ th inference result responding to $k$ th rule, and the higher value of $q_{k i}$, the higher the trust for the corresponding power and antenna setting.

The action of the $k$ th rule is chosen by an exploration/exploitation policy using $\varepsilon$-greed method as follows:

$$
c(k)=\left\{\begin{array}{ll}
\underset{i=1,2, \cdots, 9}{\operatorname{random}(i),} & \text { with prob. } \varepsilon \\
\arg \max _{i=1,2, \cdots, 9} q_{k i}, & \text { with prob. } 1-\varepsilon
\end{array} .\right.
$$

Here, the greed action factor $\varepsilon$ is decreased to zero as the optimization step increases.

The best fuzzy inference rules are obtained by using the quality function $Q^{\pi}(s, a)$ that is defined as the expected sum of discounted rewards from the initial state $s_{0}$ under policy $\pi$ as follows:

$$
Q^{\pi}(s, a)=E_{\pi}\left[\sum_{t=0}^{\infty} \gamma^{t} r\left(s_{t}, a_{t}\right) \mid s_{0}=s, a_{0}=a\right] .
$$

In Equation 19, $s_{t}$ and $a_{t}$ denote the state and the action of the fuzzy inference rule at step $t$, and $\gamma$ is the discount factor.

The Q-learning algorithm solves the quality function iteratively using the following temporal difference update:

$$
Q_{t+1}\left(s_{t}, a_{t}\right)=Q_{t}\left(s_{t}, a_{t}\right)+\Delta Q_{t}
$$

To define the incremental quality of quality function $\Delta Q$, the reward value, the quality function, and the value function are needed. The reward value is defined as the JKPI difference between recent learning steps:

$$
r_{t}=\Delta \mathrm{JKPI}=\mathrm{JKPI}_{t+1}-\mathrm{JKPI}_{t} .
$$

The quality function of the activated rules is calculated as

$$
Q_{t}\left(s_{t}, a_{t}\right)=\sum_{k} O_{k}^{(3)} q_{k c(k)}
$$

The value function of the new state after performing the applied action is calculated as:

$$
V_{t}\left(s_{t+1}\right)=\sum_{k} O_{k}^{(3)} \max _{i} q_{k i}
$$

So, using the above three parameters, $\Delta Q$ is calculated as

$$
\Delta Q_{t}=\xi\left(r_{t+1}+\gamma V_{t}\left(s_{t+1}\right)-Q_{t}\left(s_{t}, a_{t}\right)\right)
$$

and the elementary quality $q_{k i}$ should be updated by

$$
\Delta q_{k i}=\left\{\begin{array}{ll}
\xi O_{k}^{(3)} \Delta Q, & \text { if } i=c(k) \\
0, & \text { otherwise }
\end{array} .\right.
$$

In Equation 25, $\xi$ is the learning rate for Q-learning.

All distributed self-optimization entities perform the same learning function in similar conditions. So, the learning experience of any distributed entity, which is represented as the parameters of learning method, can be used by another entity. Enabling cooperative learning through sharing quality values can also improve the learning speed and the quality of the fuzzy inference rule base. To share their experience, after every learning step of Q-learning, each entity updates its quality values of the rules according to the overall shared experience. To counter for the fact that different entities may have different experiences under similar dynamics, we use the average experience over all entities and calculate it as

$$
q_{k i}^{j}=\frac{1}{M} \sum_{m=1}^{M} q_{k i}^{m}
$$

Here, $q_{k i}^{j}$ denotes the quality value recorded in selfoptimization entity $j$ corresponding to the $i$ th action of the $k$ th rule, and $M$ is the number of the SON entities managed by the central network management system (NMS).

\subsection{Reinforced parameter learning}

The reinforcement learning procedure [27] is executed to enable error back-propagation that minimizes a quadratic error function. Specifically, a reinforcement signal is propagated from the top to the bottom of the five-layered fuzzy neural network. Here, the parameters to adapt are the means and standard deviations of the fuzzification and defuzzification Gaussian membership. The quadratic error function is defined as

$$
E(t)=\frac{1}{2} e^{2}(t)=\frac{1}{2}\left(y^{*}-y(t)\right)^{2},
$$

where $y^{*}$ denotes the best overall experienced value of the JKPI, $y(t)$ denotes the measured JKPI at step $t$, and $e(t)=y^{*}-y(t)$ denotes the reinforcement signal. Consequently, as a result of the reinforcement learning, the algorithm adapts parameters of RL-FNN to minimize $E(t)$, which is equivalent to maintaining the overall JKPI at the best experienced value.

To update a parameter $Z(t)$, we calculate

$$
Z(t+1)=Z(t)-\eta \frac{\partial E}{\partial Z}
$$

Here, $\eta$ is the parameter learning rate. More specifically, using Equation 28, we update the mean and standard deviation of the defuzzication Gaussian membership function as

$$
c_{l m}^{(5)}(t+1)=c_{l m}^{(5)}(t)+\eta e(t) \frac{O_{l m}^{(4)} \sigma_{l m}^{(5)}}{\sum_{i=1}^{3} O_{l i}^{(4)} \sigma_{l i}^{(5)}}
$$




$$
\begin{aligned}
\sigma_{l m}^{(5)}(t+1)= & \sigma_{l m}^{(5)}(t) \\
& +\eta e(t) \frac{O_{l m}^{(4)} c_{l m}^{(5)} \sum_{i=1}^{3} O_{l i}^{(4)} \sigma_{l i}^{(5)}-O_{l m}^{(4)} \sum_{i=1}^{3} O_{l i}^{(4)} c_{l i}^{(5)} \sigma_{l i}^{(5)}}{\left(\sum_{i=1}^{3} O_{l i}^{(4)} \sigma_{l i}^{(5)}\right)^{2}} .
\end{aligned}
$$

Similarly, the corrections of the mean and standard deviation of the fuzzification Gaussian membership function are calculated as

$c_{i j}^{(2)}(t+1)=c_{i j}^{(2)}(t)-\eta \sum_{l=1}^{2} \sum_{m=1}^{3} \frac{\partial E(t)}{\partial O_{l m}^{(4)}} \sum_{k=1_{l m}}^{r_{l m}} \frac{\partial O_{l m}^{(4)}}{\partial O_{k}^{(3)}} \frac{\partial O_{k}^{(3)}}{\partial O_{i j}^{(2)}} \frac{\partial O_{i j}^{(2)}}{\partial c_{i j}^{(2)}}$

$\sigma_{i j}^{(2)}(t+1)=\sigma_{i j}^{(2)}(t)-\eta \sum_{l=1}^{2} \sum_{m=1}^{3} \frac{\partial E(t)}{\partial O_{l m}^{(4)}} \sum_{k=1}^{r_{l m}} \frac{\partial O_{l m}^{(4)}}{\partial O_{k}^{(3)}} \frac{\partial O_{k}^{(3)}}{\partial O_{i j}^{(2)}} \frac{\partial O_{i j}^{(2)}}{\partial \sigma_{i j}^{(2)}}$

The transmissions of reinforcement signal in each layer are as follows:

$$
\frac{\partial E(t)}{\partial O_{l m}^{(4)}}=-e(t) \frac{\sigma_{l m}^{(5)} c_{l m}^{(5)} \sum_{i=1}^{3} O_{l i}^{(4)} \sigma_{l i}^{(5)}-\sigma_{l m}^{(4)} \sum_{i=1}^{3} O_{l i}^{(4)} c_{l i}^{(5)} \sigma_{l i}^{(5)}}{\left(\sum_{i=1}^{3} O_{l i}^{(4)} \sigma_{l i}^{(5)}\right)^{2}}
$$

$$
\begin{aligned}
& \frac{\partial O_{l m}^{(4)}}{\partial O_{k}^{(3)}}= \begin{cases}1, & \text { if } O_{k}^{(3)} \in I_{l m}^{(4)} \\
0, & \text { otherwise }\end{cases} \\
& \frac{\partial O_{k}^{(3)}}{\partial O_{i j}^{(2)}}= \begin{cases}\prod_{n \neq j} O_{i n}^{(2)}, & \text { if } O_{i j}^{(2)} \in I_{k}^{(3)} \\
0, & \text { otherwise }\end{cases} \\
& \frac{\partial O_{i j}^{(2)}}{\partial c_{i j}^{(2)}}=O_{i j}^{(2)} \frac{2\left(O_{i}^{(1)}-c_{i j}^{(2)}\right)}{\left(\sigma_{i j}^{(2)}\right)^{2}} \\
& \frac{\partial O_{i j}^{(2)}}{\partial \sigma_{i j}^{(2)}}=O_{i j}^{(2)} \frac{2\left(O_{i}^{(1)}-c_{i j}^{(2)}\right)^{2}}{\left(\sigma_{i j}^{(2)}\right)^{3}}
\end{aligned}
$$

Similar to the quality values in Section 4.3, the SON entities also share their learning experience of RL-FNN membership function parameters. Again, after each learning step, each distributed entity updates the mean and standard deviation parameters according to the overall experience. The sharing of parameters can be represented as

$$
Z^{j}=\frac{1}{M} \sum_{m=1}^{M} Z^{m}
$$

Here, $Z^{j}$ denotes the vector of the mean and standard deviation parameters recorded in self-optimization entity $j$.

\section{Simulation and analysis}

The proposed approach is evaluated by system level LTE networks simulator developed in $\mathrm{c}++$. The simulation parameters and placement of transceivers (TRs) are set based on the interference-limited scenario with a hexagonal macro-cell deployment described in [23,28]. We simulate 7 three-sector cells with an inter-site distance of $500 \mathrm{~m}$. Twenty to sixty users are uniformly distributed in each cell, maintaining $35 \mathrm{~m}$ minimum distance to the base station. The user mobility is modeled with random walk with a constant speed of $3 \mathrm{~km} / \mathrm{h}$ (wrapping around is permitted). Users always have a full buffer (i.e., they have always traffic to send), and we use round robin scheduling.

The additional details of our network configuration and RL-FNN parameter settings are listed in Table 1.

For comparison, we define a reference RF configuration where all cells have the same fixed antenna tilt of $15^{\circ}$ and fixed total power of $46 \mathrm{dBm}$. This configuration was found to be the best configuration using discrete exhaustive search method for our simulated scenario $[22,23]$.

\subsection{Details on operation of RL-FNN}

In this section, we present the details of the RL-FNN algorithm and how it computes the degrees of fuzzy membership functions and does the inference for our simulation scenario.

As an example, Figure 3 shows the fuzzy membership functions acquired by reinforcement learning at optimization step 1,200 in our simulation process.

The membership functions determine which fuzzy set the input value belongs to and the degree of the membership. The shapes of the Gaussian membership functions determined by the optimized means and standard deviations will help to determine these factors in a more accurate way to improve the performance of the fuzzy neural network.

The fuzzy inference rules acquired by Q-learning without any human intervention could be regarded as the best $\mathrm{CCO}$ action policies for all states, which can hardly be intuitively set in such a complex optimization scenario, in achieving high coverage and capacity performance (see Table 2 for the complete rule base for the 81 rules generated at optimization step 1,200). For instance, according to number 35 in Table 2, when the power is medium, antenna tilt is low, traffic load compared to neighbors is high, and performance compared to neighbors is medium, the best tuning action is to make the antenna tilt higher. That is to say, the current traffic load is higher than average, and it is found to be better to make the tilt higher to shrink the coverage. Such an action may increase the performance due 
Table 1 Simulation parameters

\begin{tabular}{|c|c|}
\hline Parameter & Value \\
\hline Carrier frequency & $2.0 \mathrm{GHz}$ \\
\hline Channel bandwidth & $10 \mathrm{MHz}$ \\
\hline Propagation loss & $-128.1-37.6 \log 10(d), d$ in $\mathrm{km}$ \\
\hline Shadowing standard deviation & $8 \mathrm{~dB}$ \\
\hline Shadowing correlation distance & $50 \mathrm{~m}$ \\
\hline Shadowing correlation & Intersite, 0.5 ; intersector, 1.0 \\
\hline Penetration loss & $20 \mathrm{~dB}$ \\
\hline Thermal noise density & $-174 \mathrm{dBm} / \mathrm{Hz}$ \\
\hline BS maximum transmit power & $46 \mathrm{dBm}$ \\
\hline $\begin{array}{l}\text { Maximum antenna front-to-back } \\
\text { attenuation } A_{m}\end{array}$ & $25 \mathrm{~dB}$ \\
\hline $\begin{array}{l}\text { Maximum vertical slant angle } \\
\text { attenuation } \mathrm{SLA}_{v}\end{array}$ & $20 \mathrm{~dB}$ \\
\hline $\begin{array}{l}\text { Horizontal half-power beam width } \\
\varphi_{3 d B}\end{array}$ & $70^{\circ}$ \\
\hline $\begin{array}{l}\text { Vertical half-power beam width } \\
\theta_{3 d B}\end{array}$ & $10^{\circ}$ \\
\hline BS and UE antenna height & $32 \mathrm{~m} ; 1.5 \mathrm{~m}$ \\
\hline Power allocation on channels & Equal allocation \\
\hline $\begin{array}{l}\text { Self-optimization time } \\
\text { interval(learning time interval) }\end{array}$ & $100 \mathrm{~ms}$ \\
\hline KPI weight factor $\omega$ & 0.8 \\
\hline JKPI weight factor $\alpha$ & 0.5 \\
\hline Initial greed action factor $\varepsilon$ & 0.1 \\
\hline $\begin{array}{l}\text { Reducing rate of greed action } \\
\text { factor }\end{array}$ & $0.001 / \mathrm{s}$ \\
\hline Discount factor $\gamma$ & 0.7 \\
\hline Learning rate for Q-learning $\xi$ & 0.5 \\
\hline Parameter learning factor $\eta$ & 0.2 \\
\hline
\end{tabular}

to less inference to neighbors, more antenna gain to users in the current cell and load redistribution. However, it cannot be known which of these factors cause the highest improvement and some even may cause negative effects, but nevertheless the overall performance will improve.

\subsection{Evaluation of coverage and capacity}

In order to test the CCO performance of RL-FNN, we start the simulation with a poor configuration with very low power and very low antenna tilt, which are $8^{\circ}$ and $40 \mathrm{dBm}$, respectively. After the initialization, RL-FNN approach starts to be executed in all entities periodically to optimize the coverage and capacity performance.

Figure 4 shows the initial and the resulting SINR distributions excluding shadow fading. In Figure 4a, due to the poor initial configuration, SINR value is lower than $10 \mathrm{~dB}$ in almost the entire area which results in poor network coverage and capacity performance. As the entities
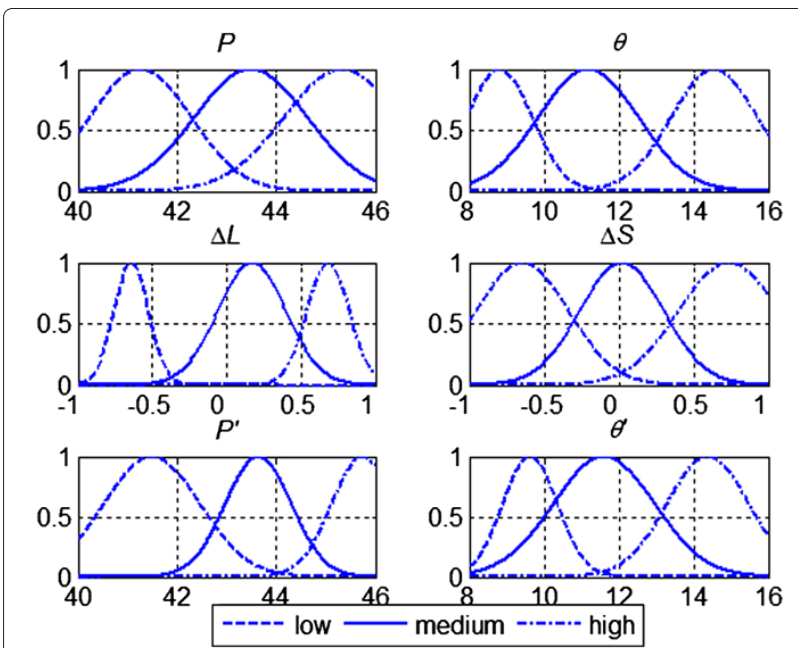

Figure 3 Membership functions acquired by reinforcement learning.

in cells learn the optimization experience cooperatively and optimize their RF configurations periodically, the SINR situation improves to be greater than $15 \mathrm{~dB}$ (see Figure 4b).

The average JKPI of overall sectors during the optimization process is shown in Figure 5. Initially, JKPI is very low and with RL-FNN improves from approximately 175 to $200 \mathrm{kbps}$ at the very beginning of the optimization. The reason for this fast improvement is that at the beginning, entities without any experience will randomly choose the fuzzy inference rules. This extends the range of configuration adjustment and avoids being limited to a local configuration space. Later, entities gradually learn and share the operation experience of which learning parameters and RF configurations may result in a better performance. In addition, the entities still execute exploration policy according to Equation 18, which allows accumulating a diverse set of experiences. However, the side effect of such explorations is the resulting temporary low JKPI. Figure 5 shows that, generally, RL-FNN gradually improves the JKPI. After about step 700, $70 \mathrm{~s}$ after the initialization, we do not see significant improvement in JKPI, and JKPI converges to around $220 \mathrm{kbps}$ although it still fluctuates slightly due to the mobility of users and dynamics of channel conditions. In addition, the JKPI is higher than that achieved by the reference which is regarded as the best configuration and was not outperformed in $[21,22]$. The improvement of JKPI comes from optimizing RF configurations according to the local traffic load distribution, which improves the resource utilization while the reference configuration cannot adapt to the traffic dynamics.

Figure 6 shows the CDF of user throughput. Compared to the initial non-optimal setting at optimization step 0 , 
Table 2 Fuzzy inference rule base acquired by Q-learning

\begin{tabular}{|c|c|c|c|c|c|c|c|c|c|c|c|c|c|}
\hline \multirow[b]{2}{*}{ Number } & \multicolumn{4}{|c|}{ If } & \multicolumn{2}{|c|}{ Then } & \multirow[b]{2}{*}{ Number } & \multicolumn{4}{|c|}{ If } & \multicolumn{2}{|c|}{ Then } \\
\hline & $P$ & $\theta$ & $\Delta L$ & $\Delta S$ & $P^{\prime}$ & $\theta^{\prime}$ & & $P$ & $\theta$ & $\Delta L$ & $\Delta S$ & $P^{\prime}$ & $\overline{\theta^{\prime}}$ \\
\hline 1 & $L$ & $L$ & L & $\mathrm{L}$ & $\mathrm{L}$ & $\mathrm{H}$ & 43 & M & $M$ & $\mathrm{H}$ & $\mathrm{L}$ & L & L \\
\hline 2 & $L$ & $L$ & L & M & M & $\mathrm{H}$ & 44 & M & M & $\mathrm{H}$ & $M$ & $\mathrm{H}$ & L \\
\hline 3 & $\mathrm{~L}$ & $L$ & L & $\mathrm{H}$ & $\mathrm{L}$ & $\mathrm{L}$ & 45 & M & M & $\mathrm{H}$ & $\mathrm{H}$ & M & L \\
\hline 4 & $L$ & $L$ & M & L & M & $\mathrm{H}$ & 46 & M & $\mathrm{H}$ & L & L & $\mathrm{H}$ & $\mathrm{H}$ \\
\hline 5,6 & $\mathrm{~L}$ & $L$ & M & $\mathrm{M}, \mathrm{H}$ & $\mathrm{L}$ & $\mathrm{H}$ & 47 & M & $\mathrm{H}$ & L & $M$ & M & $\mathrm{H}$ \\
\hline 7 & $L$ & $L$ & $\mathrm{H}$ & L & $\mathrm{H}$ & $\mathrm{H}$ & 48 & M & $\mathrm{H}$ & L & $\mathrm{H}$ & L & $\mathrm{H}$ \\
\hline 8 & $L$ & $L$ & M & $\mathrm{H}$ & M & $\mathrm{H}$ & 49,50 & M & $\mathrm{H}$ & M & $L, M$ & L & L \\
\hline 9 & $\mathrm{~L}$ & $L$ & $\mathrm{H}$ & $\mathrm{H}$ & M & $\mathrm{L}$ & 51 & M & $\mathrm{H}$ & M & $\mathrm{H}$ & $\mathrm{H}$ & L \\
\hline 10 & $\mathrm{~L}$ & M & L & $\mathrm{L}$ & $\mathrm{H}$ & $\mathrm{H}$ & 52 & M & $\mathrm{H}$ & $\mathrm{H}$ & L & L & $\mathrm{H}$ \\
\hline 11 & $L$ & M & L & M & M & $\mathrm{H}$ & 53 & M & $\mathrm{H}$ & $\mathrm{H}$ & M & M & $\mathrm{H}$ \\
\hline 12 & L & M & L & $\mathrm{H}$ & $M$ & $\mathrm{~L}$ & 54 & M & $\mathrm{H}$ & $\mathrm{H}$ & $\mathrm{H}$ & $M$ & $M$ \\
\hline 13 & $\mathrm{~L}$ & $M$ & $M$ & L & $\mathrm{L}$ & M & 55,56 & $\mathrm{H}$ & L & L & $L, M$ & L & M \\
\hline 14 & $\mathrm{~L}$ & $M$ & $M$ & $M$ & $M$ & $\mathrm{H}$ & 57 & $\mathrm{H}$ & L & L & $\mathrm{H}$ & L & L \\
\hline 15 & $L$ & M & M & $\mathrm{H}$ & $M$ & $M$ & 58 & $\mathrm{H}$ & $\mathrm{L}$ & $M$ & L & $\mathrm{H}$ & M \\
\hline 16 & $L$ & M & $\mathrm{H}$ & L & $\mathrm{L}$ & $\mathrm{H}$ & 59 & $\mathrm{H}$ & L & $M$ & M & $M$ & $M$ \\
\hline 17,18 & $L$ & $M$ & $\mathrm{H}$ & $\mathrm{M}, \mathrm{H}$ & $\mathrm{L}$ & $M$ & 60 & $\mathrm{H}$ & $\mathrm{L}$ & M & $\mathrm{H}$ & $\mathrm{L}$ & M \\
\hline 19 & $L$ & $\mathrm{H}$ & L & L & M & $\mathrm{H}$ & 61 & $\mathrm{H}$ & $\mathrm{L}$ & $\mathrm{H}$ & $\mathrm{L}$ & L & M \\
\hline 20 & $\mathrm{~L}$ & $\mathrm{H}$ & L & $M$ & $\mathrm{~L}$ & $\mathrm{H}$ & 62 & $\mathrm{H}$ & $\mathrm{L}$ & $\mathrm{H}$ & $M$ & $\mathrm{H}$ & L \\
\hline 21 & $L$ & $\mathrm{H}$ & L & $\mathrm{H}$ & $\mathrm{L}$ & $M$ & 63 & $\mathrm{H}$ & $\mathrm{L}$ & $\mathrm{H}$ & $\mathrm{H}$ & M & M \\
\hline 22 & $\mathrm{~L}$ & $\mathrm{H}$ & $M$ & L & $M$ & $\mathrm{H}$ & 64,65 & $\mathrm{H}$ & $M$ & L & $L, M$ & $\mathrm{~L}$ & $M$ \\
\hline 23 & $L$ & $\mathrm{H}$ & $M$ & $M$ & $M$ & M & 66 & $\mathrm{H}$ & M & L & $\mathrm{H}$ & $\mathrm{H}$ & M \\
\hline 24 & $\mathrm{~L}$ & $\mathrm{H}$ & $M$ & $\mathrm{H}$ & $M$ & $\mathrm{H}$ & 67 & $\mathrm{H}$ & $M$ & $M$ & L & $M$ & $\mathrm{H}$ \\
\hline 25,26 & $\mathrm{~L}$ & $\mathrm{H}$ & $\mathrm{H}$ & $L, M$ & $\mathrm{H}$ & $M$ & 68 & $\mathrm{H}$ & $M$ & $M$ & M & $M$ & $M$ \\
\hline 27 & L & $\mathrm{H}$ & $\mathrm{H}$ & $\mathrm{H}$ & $L$ & L & 69 & $\mathrm{H}$ & M & $M$ & $\mathrm{H}$ & $\mathrm{H}$ & $\mathrm{H}$ \\
\hline 28 & M & $L$ & L & L & $M$ & $M$ & 70 & $\mathrm{H}$ & $M$ & $\mathrm{H}$ & L & $\mathrm{L}$ & $\mathrm{H}$ \\
\hline 29 & M & $L$ & L & $M$ & $\mathrm{H}$ & $\mathrm{L}$ & 71 & $\mathrm{H}$ & $M$ & $\mathrm{H}$ & M & L & M \\
\hline 30 & M & $L$ & L & $\mathrm{H}$ & $\mathrm{L}$ & $\mathrm{L}$ & 72 & $\mathrm{H}$ & $M$ & $\mathrm{H}$ & $\mathrm{H}$ & $M$ & M \\
\hline 31,32 & $M$ & $L$ & $M$ & $L, M$ & $M$ & $M$ & 73 & $\mathrm{H}$ & $\mathrm{H}$ & L & L & $\mathrm{H}$ & M \\
\hline 33 & M & $L$ & M & $\mathrm{H}$ & $M$ & $\mathrm{~L}$ & 74,75 & $\mathrm{H}$ & $\mathrm{H}$ & L & $\mathrm{M}, \mathrm{H}$ & M & M \\
\hline 34 & M & $L$ & $\mathrm{H}$ & L & $M$ & $\mathrm{H}$ & 76 & $\mathrm{H}$ & $\mathrm{H}$ & $M$ & L & $\mathrm{L}$ & $\mathrm{L}$ \\
\hline 35 & M & $L$ & $\mathrm{H}$ & $M$ & $M$ & $M$ & 77 & $\mathrm{H}$ & $\mathrm{H}$ & M & $M$ & $M$ & $\mathrm{H}$ \\
\hline 36 & $M$ & $L$ & $\mathrm{H}$ & $\mathrm{H}$ & $\mathrm{H}$ & $M$ & 78 & $\mathrm{H}$ & $\mathrm{H}$ & $M$ & $\mathrm{H}$ & $\mathrm{H}$ & L \\
\hline 37,38 & M & M & L & $L, M$ & $\mathrm{~L}$ & $\mathrm{H}$ & 79 & $\mathrm{H}$ & $\mathrm{H}$ & $\mathrm{H}$ & L & L & $\mathrm{H}$ \\
\hline 39 & M & M & L & $\mathrm{H}$ & $\mathrm{L}$ & $\mathrm{H}$ & 80 & $\mathrm{H}$ & $\mathrm{H}$ & $\mathrm{H}$ & $M$ & $\mathrm{~L}$ & M \\
\hline 40 & $M$ & $M$ & M & L & $\mathrm{H}$ & M & 81 & $\mathrm{H}$ & $\mathrm{H}$ & $\mathrm{H}$ & $\mathrm{H}$ & $\mathrm{H}$ & L \\
\hline 41,42 & M & M & M & $\mathrm{M}, \mathrm{H}$ & $\mathrm{L}$ & $M$ & & & & & & & \\
\hline
\end{tabular}

the coverage (i.e., $5 \%$ tile of user throughput) and capacity (i.e., $50 \%$ tile of user throughput) performance of RLFNN are improved by $19.5 \%$ and $48.9 \%$. Compared to the reference configuration, the coverage and capacity performance are also improved by $7.9 \%$ and $1.5 \%$. However, from the CDF figure, we can also see that the reference is able to achieve higher throughput values and RL-FNN may decrease the performance of high-throughput users. Note that RL-FNN optimizes power and antenna beam orientation with the goal of improving the 5\% tile and 50\% tile throughput performance. Nevertheless, we believe this degradation of high-throughput users is acceptable because the fairness across users is improved with significant improvement at the cell-edge. 


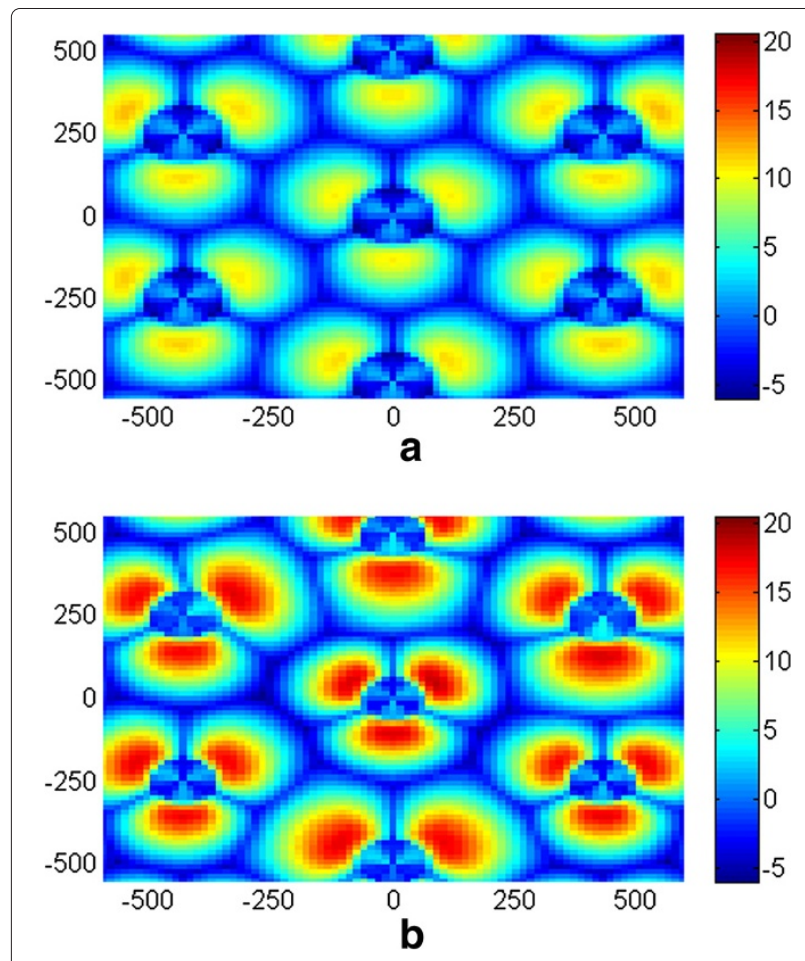

Figure 4 Simulated network SINR distribution excluding shadow fading. (a) To be optimized (step 0). (b) Optimized (step 1,200).

\subsection{Additional benefits: energy efficiency}

Adapting total power may also improve overall energy efficiency in addition to coverage and capacity. Figures 7 and 8 show the average energy consumption and the average energy efficiency, respectively, during optimization process. We compute energy efficiency as throughput per watt. It is evident that the energy consumption of RL-FNN is much lower and the energy efficiency is much higher than the reference.

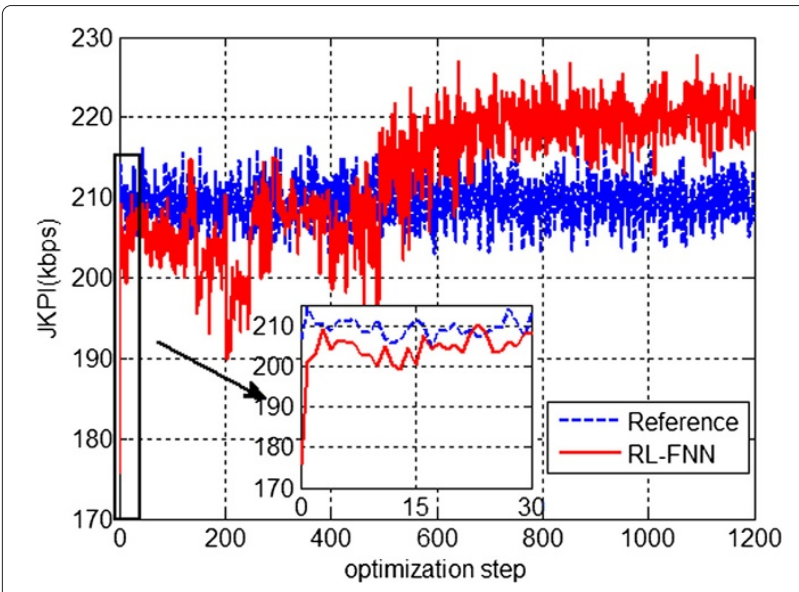

Figure 5 Average JKPI.

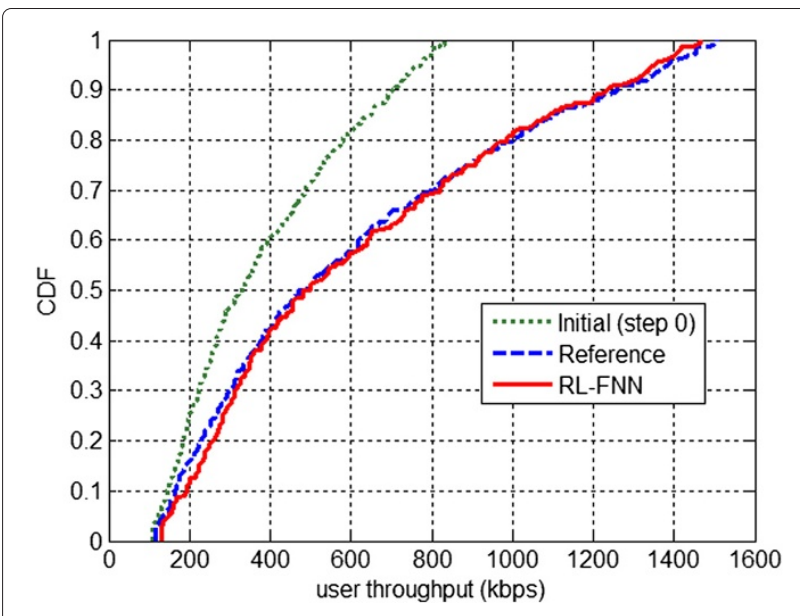

Figure 6 CDF of user throughput.

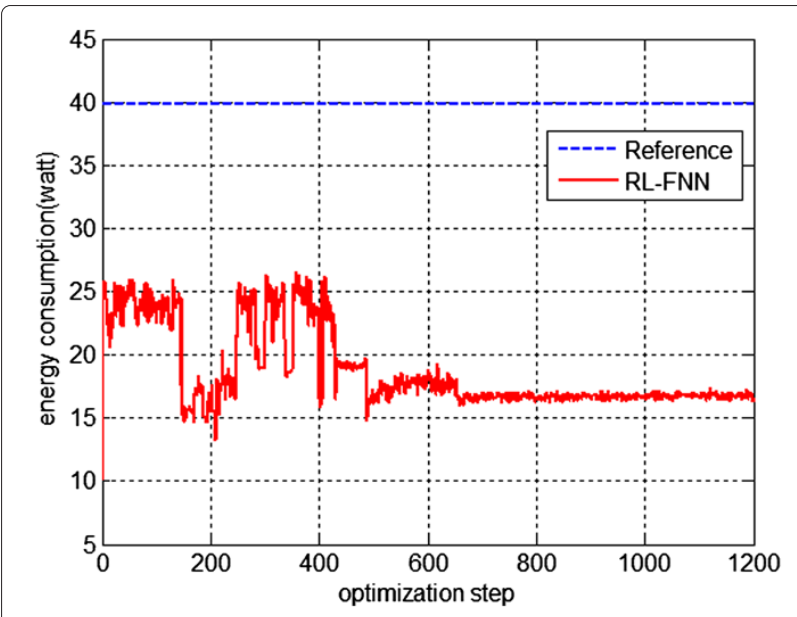

Figure 7 Average energy consumption.

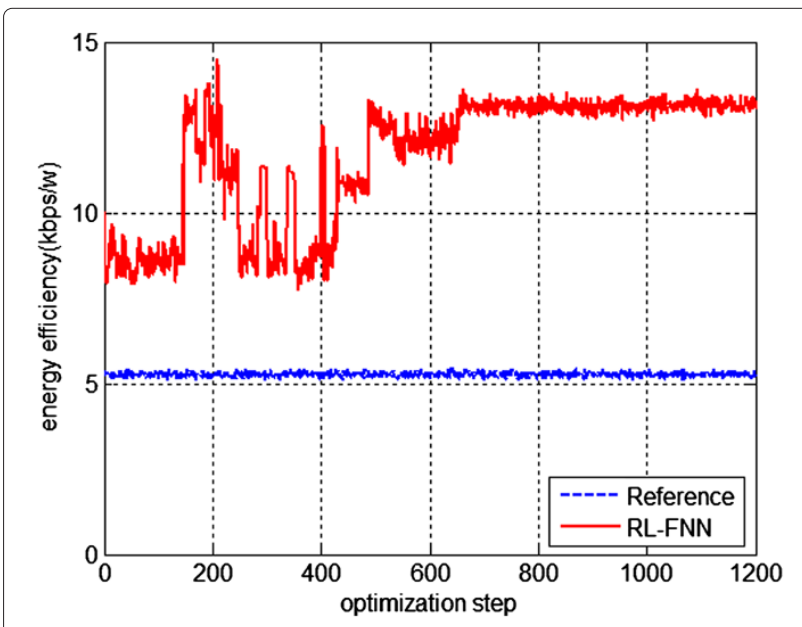

Figure 8 Average energy efficiency. 
Table 3 Performance comparison

\begin{tabular}{lccc}
\hline Indicator & RL-FNN & Reference & $\begin{array}{c}\text { Improvement } \\
\text { (\%) }\end{array}$ \\
\hline JKPI & $219.6 \mathrm{kbps}$ & $209.2 \mathrm{kbps}$ & 5.0 \\
Energy consumption & $42.3 \mathrm{dBm}(16.8 \mathrm{~W})$ & $46 \mathrm{dBm}(39.8 \mathrm{~W})$ & 57.8 \\
Energy efficiency & $13.1 \mathrm{kbps} / \mathrm{W}$ & $5.26 \mathrm{kbps} / \mathrm{W}$ & 147.1 \\
\hline
\end{tabular}

The average JKPI, energy consumption, and energy efficiency comparison of RL-FNN and the reference after step 700 are listed in Table 3. RL-FNN can improve coverage and capacity by $5 \%$ compared to the best configuration found with exhaustive search but improves energy consumption by $57.8 \%$, and energy efficiency improves by $147.1 \%$. The huge improvement comes from the optimized RF configuration. On one hand, lower interference will be caused by cell-edge users of neighboring cells resulting from lower power. On the other hand, the coverage of cells is optimized considering the traffic distribution, and the antenna beam orientation is optimized to mainly serve UE traffic within the coverage area while minimizing the interference to other cells. Although the power is lower than the maximum setting, it is better utilized with the help of the optimized directed antenna beam.
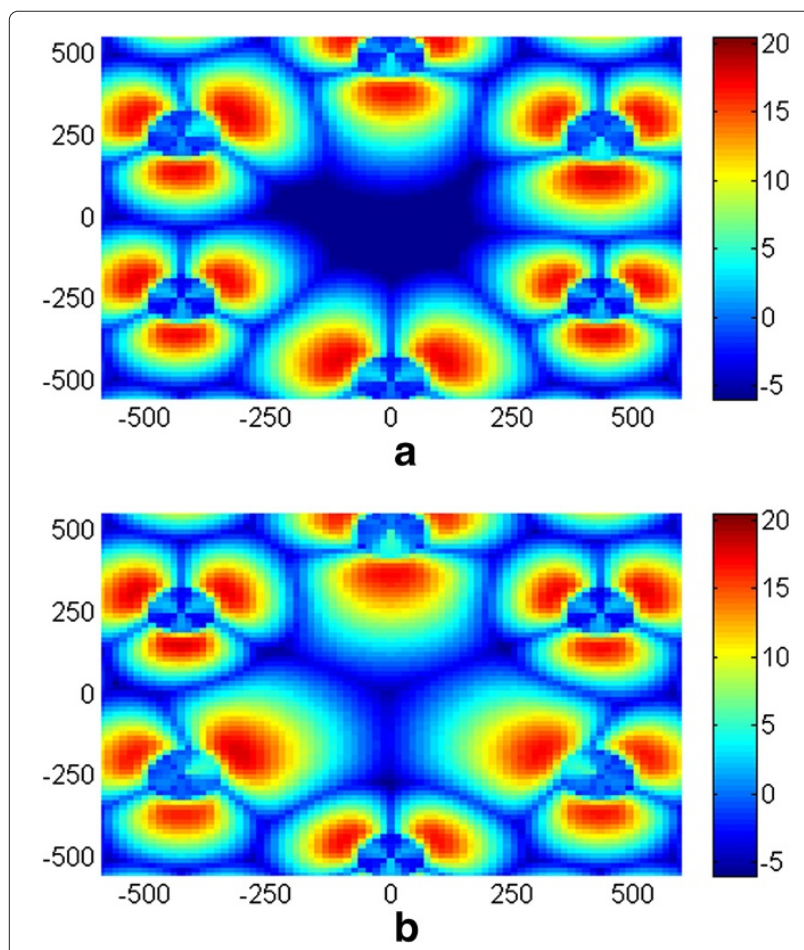

Figure 9 Simulated network SINR distribution excluding shadow fading under abrupt changes. (a) To be optimized (step 1,201). (b) Optimized (step 2,400).

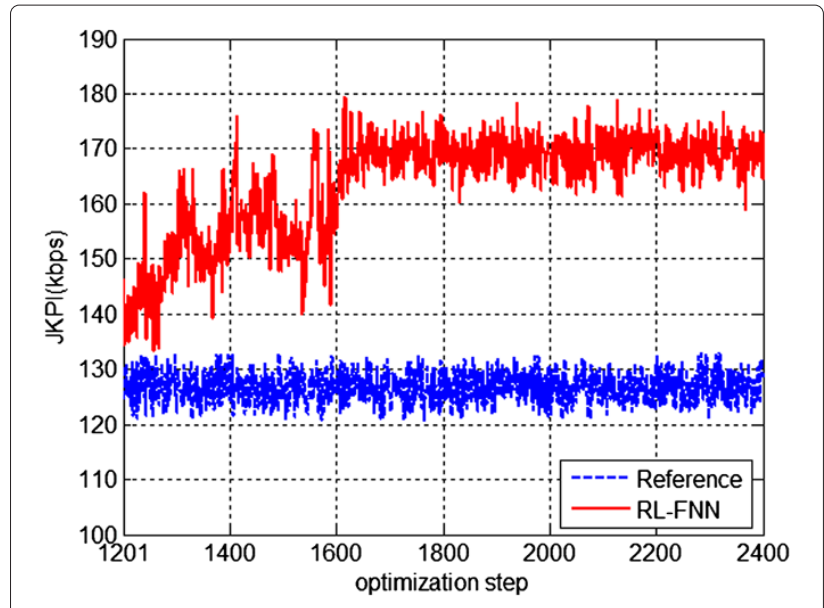

Figure 10 Average JKPI under abrupt changes.

The above simulation results demonstrate that the proposed RL-FNN approach is able to achieve high performance in terms of coverage and capacity with significantly lower energy consumption.

\subsection{Performance under abrupt changes}

In this section, we evaluate the performance of RL-FNN when a cell is shut down due to, for instance, an unexpected failure or for simply energy-saving purposes. In the simulation, a cell is shut down at the following step 1,201 and all entities execute RL-FNN approach periodically to optimize the coverage and capacity performance.

Figure 9 shows the SINR distribution comparison of the initial environment at step 1,201 and the optimized. When a cell is shut down, a coverage hole with a SINR of lower than $-8 \mathrm{~dB}$ occurs which needs to be covered by the neighboring cells. This will consequently affect the

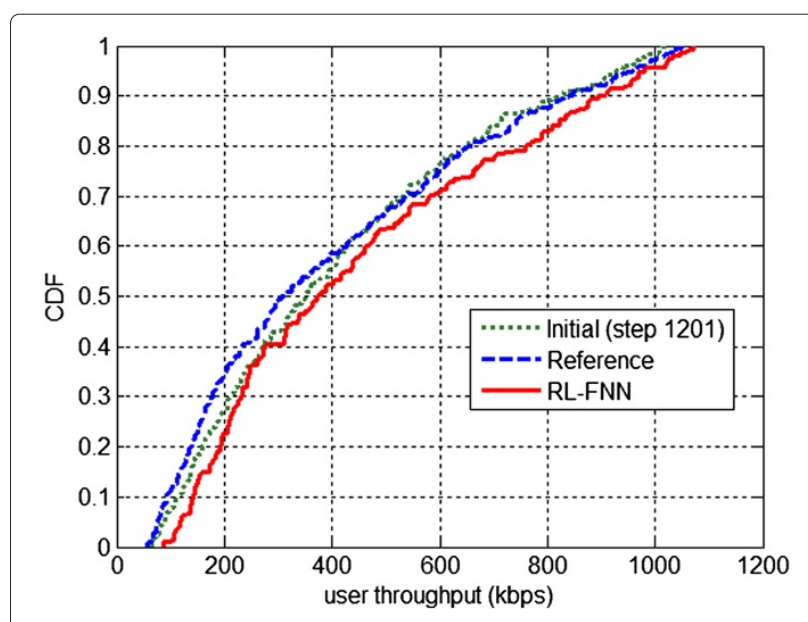

Figure $11 \mathrm{CDF}$ of user throughput under abrupt changes. 

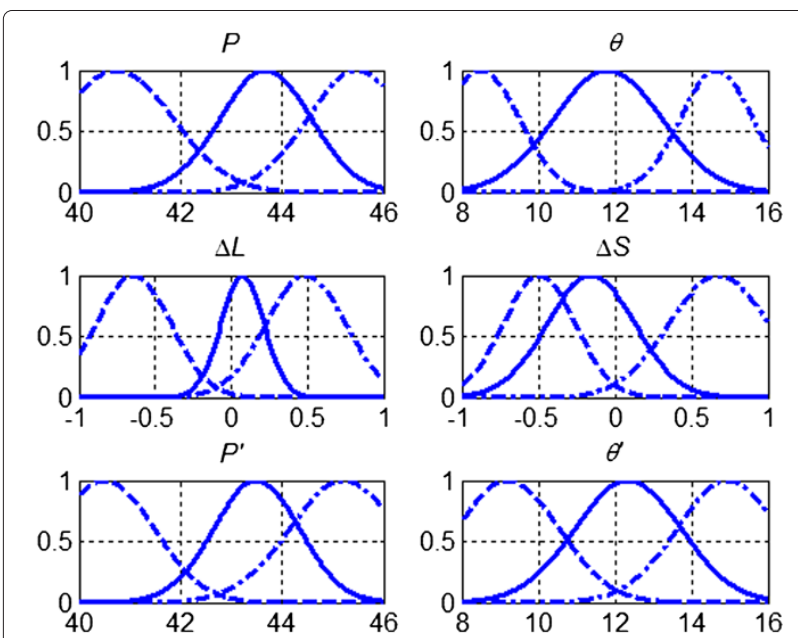

Figure 12 Modified membership functions.

traffic load distribution of the neighboring cells. After the RF configurations are optimized, the hole is covered by neighboring cells with lower antenna tilts and the average SINR value in that area improves to $6.71 \mathrm{~dB}$.

The variations of average JKPI of sectors nearby the coverage hole during optimization process after shutting down one cell are presented in Figure 10. At step 1,201, some of the users of the shut-down cell will be located in the coverage hole area and hence become the cell-edge users for the neighboring cells. This consequently worsens the cell-edge performance of those cells. So, the JKPI resulting from the RF configurations at step 1,201 is low but still higher than the reference due to the optimized
RF configurations in the previous optimization process. Later, the RL-FNN gradually improves the JKPI by adapting the RF configurations to the new situation. At about step 1,700, $50 \mathrm{~s}$ after shutting down the cell, cooperative learning process converges with JKPI approximately around $170 \mathrm{kbps}$. There is no significant improvement after step 1,700 .

Figure 11 shows the throughput $\mathrm{CDF}$ of the users in the coverage hole area and in the sectors nearby the coverage hole. Compared to performance at step 1,201, the coverage and capacity performance of RL-FNN are improved by $33.4 \%$ and $7.0 \%$, respectively. Compared to the reference configuration, the coverage and capacity performance are improved by $53.2 \%$ and $21.3 \%$, respectively, achieving significant improvements.

The adapted fuzzy membership functions and fuzzy inference rules in the coverage hole scenario at step 2,400 are shown in Figure 12 and Table 4. There is a little difference between the optimized shapes of the membership functions and those at step 1,200. The modifications of the membership functions are adapted to the coverage hole scenario and will help to determine the degree of membership function in a better way to improve the performance. Also, some of the fuzzy inference rules are modified. Compared to the old rules, we can see that entities become more likely to provide more power with lower antenna tilt to respond to the coverage hole. Take rule number 34 for example, when the power is medium, antennae tilt is low, traffic load compared to neighbors is high, and performance compared to neighbors is low, the best tuning action is to make the power higher while keeping low antenna tilt. That is to say, if a sector covers some users

Table 4 Modified fuzzy inference rules

\begin{tabular}{|c|c|c|c|c|c|c|}
\hline \multirow[b]{2}{*}{ Number } & \multicolumn{4}{|c|}{ If } & \multicolumn{2}{|c|}{ Then } \\
\hline & $P$ & $\theta$ & $\Delta L$ & $\Delta S$ & $P^{\prime}$ & $\theta^{\prime}$ \\
\hline 14,15 & $L$ & $M$ & $M$ & $\mathrm{M}, \mathrm{H}$ & $\mathrm{H}$ & $\mathrm{L}$ \\
\hline 18 & $L$ & M & $\mathrm{H}$ & $\mathrm{H}$ & M & M \\
\hline 20,21 & $L$ & $\mathrm{H}$ & $L$ & $\mathrm{M}, \mathrm{H}$ & $\mathrm{H}$ & $\mathrm{H}$ \\
\hline 23,24 & $L$ & $\mathrm{H}$ & $M$ & $\mathrm{M}, \mathrm{H}$ & $L$ & M \\
\hline 34 & M & $\mathrm{L}$ & $\mathrm{H}$ & L & $\mathrm{H}$ & M \\
\hline 35 & M & $L$ & $\mathrm{H}$ & M & $\mathrm{H}$ & M \\
\hline 39 & M & M & $\mathrm{L}$ & $\mathrm{H}$ & M & $\mathrm{H}$ \\
\hline 42 & M & M & $M$ & $\mathrm{H}$ & $\mathrm{H}$ & M \\
\hline 43 & M & M & $\mathrm{H}$ & L & $\mathrm{H}$ & M \\
\hline 45 & M & M & $\mathrm{H}$ & $\mathrm{H}$ & M & $\mathrm{H}$ \\
\hline 50 & M & $\mathrm{H}$ & $M$ & M & $\mathrm{H}$ & L \\
\hline 69 & $\mathrm{H}$ & M & $M$ & $\mathrm{H}$ & M & $\mathrm{H}$ \\
\hline $70,71,72$ & $\mathrm{H}$ & M & $\mathrm{H}$ & $L, M, H$ & $\mathrm{H}$ & L \\
\hline 78 & $\mathrm{H}$ & $\mathrm{H}$ & $M$ & $\mathrm{H}$ & $\mathrm{H}$ & $M$ \\
\hline
\end{tabular}




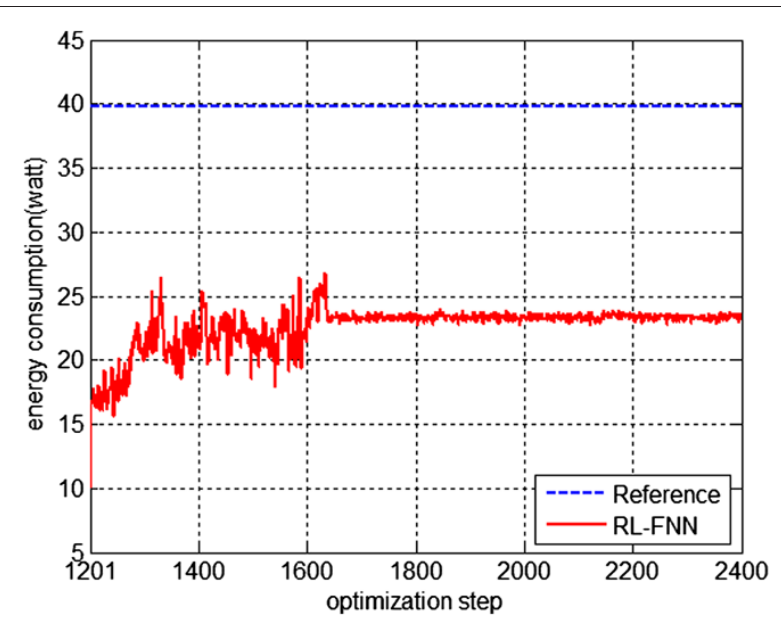

Figure 13 Average energy consumption under abrupt changes.

within the hole which will increase the traffic load and lower the performance, it is better for this sector to make the power higher in improving the performance. Consequently, potentially, more users within the hole can be covered by this sector. Another example is rule number 39 : when the power is medium, antennae tilt is medium, traffic load compared to neighbors is low, and performance compared to neighbors is high, the best tuning action is to make the antenna tilt higher. This rule will mainly apply to the sector which is nearby the hole but cannot cover many users within the hole due to its antenna beam orientation. So it is better for this sector to make the antenna tilt higher which will lower the interference to users within the hole. In summary, different rules are acquired for different situations, and the acquired rule base helps to improve the overall performance in the dynamic environment.

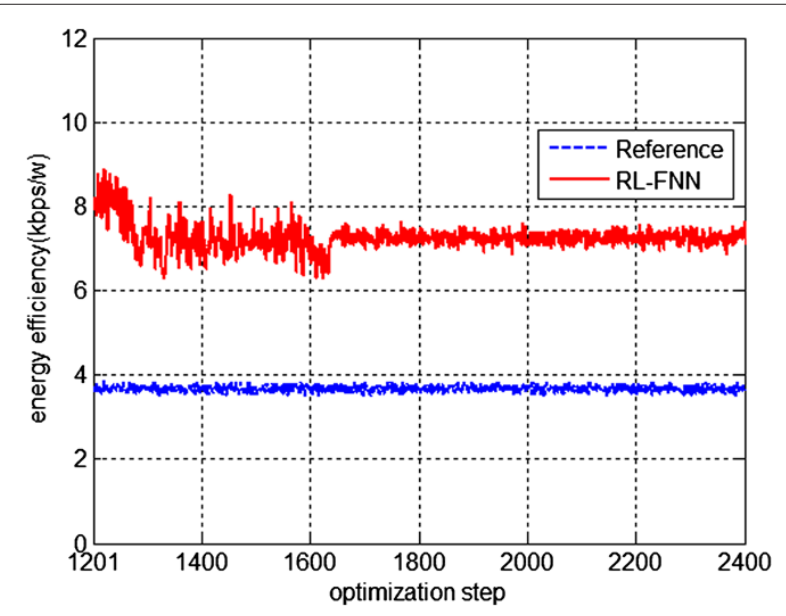

Figure 14 Average energy efficiency under abrupt changes.
Table 5 Performance comparison under abrupt changes

\begin{tabular}{lccc}
\hline Indicator & RL-FNN & Reference & $\begin{array}{c}\text { Improvement } \\
\text { (\%) }\end{array}$ \\
\hline JKPI & $169.5 \mathrm{kbps}$ & $126.8 \mathrm{kbps}$ & 33.7 \\
Energy consumption & $43.7 \mathrm{dBm}(23.2 \mathrm{~W})$ & $46 \mathrm{dBm}(39.8 \mathrm{~W})$ & 41.7 \\
Energy efficiency & $7.3 \mathrm{kbps} / \mathrm{W}$ & $3.2 \mathrm{kbps} / \mathrm{W}$ & 128.1 \\
\hline
\end{tabular}

Additionally, Figures 13 and 14, respectively, show the average energy consumption and the average energy efficiency during optimization process. It is evident that the energy consumption of RL-FNN is still lower and the energy efficiency is much higher than the reference in such a coverage hole scenario.

The average JKPI, energy consumption, and energy efficiency comparison of RL-FNN and the reference after step 1,700 are listed in Table 5. RL-FNN significantly improves coverage and capacity by $33.7 \%$. Energy consumption is again low, with an improvement of $41.7 \%$ and energy efficiency improves $128.1 \%$. Note that in this scenario, some of the neighboring cells lower their antenna tilts to cover the users located in the coverage hole. Therefore, more power than the initial step will be used due to these adaptations. Still the power is lower than the maximum constraint and is better utilized.

In summary, the simulation results demonstrate that the proposed RL-FNN approach can efficiently improve the coverage and capacity performance in a dynamic environment. The results show that in approximately 700 to 800 optimization steps, RL-FNN converges to a better setting than the reference from an initially badly chosen configuration. Also, in 500 to 600 steps, RL-FNN recovers from the coverage hole scenario. However, note that the actual time for reaching these states is determined by the time interval for the cell to collect the load and spectrum efficiency indicator of neighbors and to perform the adjustments. Given these considerations, we assume that the optimization step interval is approximately $0.1 \mathrm{~s}$ as our approach can operate with low granularity. Hence, we expect that RL-FNN can operate with convergence times in the order of a minute. Compared to $[9,21,22]$ which need nearly 1,000 optimization steps for the onedimensional optimization of antenna tilt, the convergence rate of RL-FNN is a significant improvement as it adjusts both power and antenna tilt. Such fast convergence rate can only be achieved by cooperative learning enabled by RL-FNN.

\section{Conclusions}

In this paper, an online approach has been presented for self-optimization of coverage and capacity in LTE networks. The proposed RL-FNN approach is based on the fuzzy neural network combined with Q-learning and reinforced parameter learning. All self-optimization entities 
operate in a distributed manner and try to optimize power and antenna tilt automatically and cooperatively using the shared optimization experience.

From the simulation results, we conclude that our approach is able to acquire robust optimization policies for different complex scenarios and maintains a significantly better performance in terms of coverage and capacity with low energy consumption. This especially results in a dramatic improvement in energy efficiency. Finally, RL-FNN converges with an acceptable rate and is therefore applicable to different dynamic scenarios and applications.

In our future work, variants of the algorithms will be developed to enhance the cooperation between SON entities especially when abrupt changes happen. Moreover, it would be an interesting future research to extend the current work to heterogeneous networks.

\section{Competing interests}

The authors declare that they have no competing interests.

\section{Acknowledgements}

This work was supported by the National Natural Science Foundation of China (No. 61231009), National Major Science and Technology Special Project of China (No. 2013ZX03003016), National High Technology Research and Development Program of China (863 Program) (No. 2014AA01A705), and Funds for Creative Research Groups of China (No. 61121001).

\section{Author details \\ ${ }^{1}$ State Key Laboratory of Networking and Switching Technology, Beijing University of Posts and Telecommunications, Beijing 100876, China. 2 Department of Computing and Communication Technologies, Oxford Brookes University, Wheatley, Oxford OX33 1HX, UK.}

Received: 5 September 2013 Accepted: 3 April 2014 Published: 12 April 2014

\section{References}

1. ON ON Yilmaz, J Hämäläinen, S Hämäläinen, Optimization of adaptive antenna system parameters in self-organizing LTE networks. Wireless Netw. 19(6), 1251-1267 (2013)

2. 4G Americas, White Paper: Self-optimizing networks: the benefits of SON in LTE (2013). http://www.4gamericas.org. Accessed on 7 October

3. SOCRATES: Self-optimisation and self-configuration in wireless networks. http://www.fp7-socrates.eu.org. Accessed on 6-8 February 2008

4. 3GPP TS 32.500 Vb.1.0, Self-organizing networks (SON); Concepts and requirements. http://www.3gpp.org. Accessed on 22 December 2011

5. 3GPP TR 36.902 V9.3.1, E-UTRA; Self-configuring and self-optimizing network (SON) use cases and solutions. http://www.3gpp.org. Accessed on 7 April 2011

6. 3GPP TS 32.541 Vb.0.0: SON; Self-healing concepts and requirements. http://www.3gpp.org. Accessed on 7 April 2011

7. D Lopez-Perez, X Chu, AV Vasilakos, H Claussen, On distributed and coordinated resource allocation for interference mitigation in self-organizing LTE networks. IEEE/ACM Trans. Netw. 21(4), 1145-1158 (2013)

8. S Sengupta, S Das, M Nasir, AV Vasilakos, W Pedrycz, An evolutionary multiobjective sleep-scheduling scheme for differentiated coverage in wireless sensor networks. Syst. Man Cybernet. Part C: Appl. Rev. IEEE Trans. 42(6), 1093-1102 (2012)

9. J Li, J Zeng, X Su, W Luo, J Wang, Self-optimization of coverage and capacity in LTE networks based on central control and decentralized fuzzy Q-learning. Int. J. Distributed Sensor Netw. 2012 (2012)

10. H Eckhardt, S Klein, M Gruber, Vertical antenna tilt optimization for LTE base stations, in Vehicular Technology Conference (VTC Spring) 2011 IEEE 73rd (Yokohama, May 2011), pp. 1-5
11. I Siomina, P Varbrand, D Yuan, Automated optimization of service coverage and base station antenna configuration in UMTS networks. Wireless Commun. IEEE. 13(6), 16-25 (2006)

12. T Cai, GP Koudouridis, C Qvarfordt, J Johansson, P Legg, Coverage and capacity optimization in E-UTRAN based on central coordination and distributed Gibbs sampling, in Vehicular Technology Conference (VTC 2010-Spring), 2010 IEEE 71st, Taipei (16-19, May 2010), pp. 1-5

13. A Awada, B Wegmann, I Viering, A Klein, Optimizing the radio network parameters of the long term evolution system using Taguchi's method. Vehicular Technol. IEEE Trans. 60(8), 3825-3839 (2011)

14. Y Zeng, K Xiang, D Li, AV Vasilakos, Directional routing and scheduling for green vehicular delay tolerant networks. Wireless Netw. 19(2), 161-173 (2013)

15. AV Vasilakos, Gl Papadimitriou, A new approach to the design of reinforcement schemes for learning automata: stochastic estimator learning algorithm. Neurocomputing. 7(3), 275-297 (1995)

16. KC Zikidis, AV Vasilakos, ASAFES2: A novel, neuro-fuzzy architecture for fuzzy computing, based on functional reasoning. Fuzzy Sets Syst. 83(1), 63-84 (1996)

17. MA Khan, H Tembine, AV Vasilakos, Game dynamics and cost of learning in heterogeneous $4 \mathrm{G}$ networks. Select. Areas Commun. IEEE J. 30(1), 198-213 (2012)

18. ON Yilmaz, J Hamalainen, S Hamalainen, Self-optimization of remote electrical tilt, in Personal Indoor and Mobile Radio Communications (PIMRC) 2010 IEEE 21st International Symposium On (Instanbul, September 2010), pp. 1128-1132

19. R Razavi, S Klein, H Claussen, Self-optimization of capacity and coverage in LTE networks using a fuzzy reinforcement learning approach, in Personal Indoor and Mobile Radio Communications (PIMRC) 2010 IEEE 21st International Symposium On (Instanbul, 26-30 September 2010), pp. $1865-1870$

20. R Razavi, S Klein, H Claussen, A fuzzy reinforcement learning approach for self-optimization of coverage in LTE networks. Bell Labs Tech. J. 15(3), 153-175 (2010)

21. M Naseer ul, A Mitschele-Thiel, Reinforcement learning strategies for self-organized coverage and capacity optimization, in Wireless Communications and Networking Conference (WCNC) 2012 IEEE (Shanghai, 1-4 April 2012), pp. 2818-2823

22. M Naseer ul, A Mitschele-Thiel, Cooperative fuzzy Q-learning for self-organized coverage and capacity optimization, in Personal Indoor and Mobile Radio Communications (PIMRC) 2012 IEEE 23rd International Symposium On (Sydney, NSW, 9-12 September 2012), pp. 1406-1411

23. 3GPP TR 36.814 V9.0.0, Evolved universal terrestrial radio access (E-UTRA); Further advancements for E-UTRA physical layer aspects. http://www.3gpp.org. Accessed on 30 March 2010

24. SW Tung, C Quek, C Guan, Sohyfis-yager: A self-organizing Yager based hybrid neural fuzzy inference system. Expert Syst. Appl. 39(17), 12759-12771 (2012)

25. WX Shi, SS Fan, N Wang, CJ Xia, Fuzzy neural network based access selection algorithm in heterogeneous wireless networks. J. China Inst. Commun. 31(9), 151-156 (2010)

26. RS Sutton, AG Barto, Reinforcement Learning: An Introduction. (MIT Press Cambridge, 1998)

27. L Giupponi, Agustí R, J Pérez-Romero, O Sallent, Fuzzy neural control for economic-driven radio resource management in beyond $3 \mathrm{G}$ networks. Syst. Man, Cybernet. Part C: Appl. Rev. IEEETrans. 39(2), 170-189 (2009)

28. 3GPP TR 25.814 V7.1.0, Physical layer aspect for evolved universal terrestrial radio access (UTRA). http://www.3gpp.org. Accessed on 13 Octorber 2006

doi:10.1186/1687-1499-2014-57

Cite this article as: Fan et al: Self-optimization of coverage and capacity based on a fuzzy neural network with cooperative reinforcement learning. EURASIP Journal on Wireless Communications and Networking 2014 2014:57. 